\title{
Acute myocardial infarction with cardiogenic shock in a young physically active physician concurrently using the anabolic steroid sustanon: A case report
}

\author{
NASR ALRABADI ${ }^{1}$, MOHAMAD ISMAIL JARRAH $^{2}$ and KAREM HASAN ALZOUBI ${ }^{3}$ \\ ${ }^{1}$ Department of Pharmacology, Faculty of Medicine; ${ }^{2}$ Department of Internal Medicine, \\ Interventional Cardiology Division, Faculty of Medicine; ${ }^{3}$ Department of Clinical Pharmacy, \\ Faculty of Pharmacy, Jordan University of Science and Technology, Irbid 22110, Jordan
}

Received October 31, 2019; Accepted February 5, 2020

DOI: $10.3892 /$ br.2020.1321

\begin{abstract}
The association between ischemic heart disease (IHD) and the concurrent use of anabolic androgenic steroids (AASs) is underestimated in clinical settings. The tendency of patients to not disclose AASs use may explain this underestimation. In the present case report, the clinical case of a 26-year-old physically active male, who was a physician, without any classical coronary risk factors, who presented with chest pain that was misdiagnosed by the peripheral care unit as skeletal muscle pain is described. Later, the patient was brought to our central hospital (King Abdullah University Hospital) suffering from a massive acute myocardial infarction with marked ECG changes and cardiogenic shock. Following stabilization of his condition, a detailed history of the patient was taken, during which the patient admitted that he was a chronic user of the anabolic steroid sustanon $(250 \mathrm{mg}$, once/week for 6 months) and amino acid supplements (whey protein isolate, 6 tabs every day for 1 year). Specific cardiac markers were increased and the patient exhibited dynamic ischemic changes in his electrocardiogram. Notably, the coronary angiogram of the patient demonstrated ostial occlusion of the left anterior descending artery, which was associated with mid-right coronary artery embolic obstruction. Other than the anabolic steroids and protein supplementation use, the patient history, examination and lab evaluation were normal. During follow up, the patient continued to suffer heart failure
\end{abstract}

Correspondence to: Dr Nasr Alrabadi, Department of Pharmacology, Faculty of Medicine, Jordan University of Science and Technology, Petra Street, Irbid 22110, Jordan

E-mail: nnalrabadi@just.edu.jo

Dr Mohamad Ismail Jarrah, Department of Internal Medicine, Interventional Cardiology Division, Faculty of Medicine, Jordan University of Science and Technology, Petra Street, Irbid 22110, Jordan E-mail: mijarrah@just.edu.jo

Key words: acute myocardial infarction, cardiogenic shock, anabolic steroids, thrombosis, sustanon with low ejection fraction. In addition, he developed apical thrombus 2 months after primary admission. The patient developed tachycardia in spite of optimal medical treatment and finally received an implantable cardioverter defibrillator. Physicians should always be aware of the possibility of AASs use in young physically active patients. IHD should always be suspected and investigated with typical chest pain in healthy young patients, even if regular risk factors are not present. Medical professionals should not be excluded as potential AASs users/abusers.

\section{Introduction}

Anabolic steroids are synthetic derivatives of the naturally occurring testosterone, which can be useful in the treatment of some clinical conditions including hypogonadism. Unfortunately, these agents are often illegally or used without prescription by athletes and young physically active individuals aiming to increase their muscle mass and physical performance (1). At high doses, termed supraphysiologic or toxic doses, the use of anabolic adrenergic steroids (AASs) can predispose individuals to serious complications, which may affect a number of organs, including: the liver, kidney, sexual and reproductive organs, the brain, the neurohormonal axis and the cardiovascular system (2-4).

The association between ischemic heart disease (IHD) and the concurrent use of AASs is underestimated in medical literature, possibly due to the tendency of the affected individuals to conceal their use of AAS (5). However, several cardiovascular complications have been reported with supraphysiologic doses of anabolic steroids. Myocardial infarction, hypertension, stroke, cardiomyopathy, pulmonary embolism and fatal and non-fatal arrhythmias are examples of those complications $(2,3)$. Notably, only few case reports identified in the literature describing young AASs users suffering from acute myocardial infarction (AMI) (5). However, none of the previous reports described such a case in a young physically active physician.

The aim of the present report was to increase awareness amongst physicians concerning the use of anabolic steroids and their possible cardiovascular consequences, particularly in 
young patients without any obvious or normal cardiovascular risk factors. In addition, young medical personnel cannot be excluded from the group of potential users of non-prescription AASs. In fact, among these patients, admitting to the use of AASs may be more embarrassing compared with others.

\section{Case report}

The publication of this report was approved by the local Institutional Review Board of King Abdullah University Hospital affiliated with the Jordan University of Science and Technology (approval no. 781182018). The requirement for patient consent was waived.

A 26-year-old physically active male who had no prior medical history was admitted to the emergency department (ED) in an associated public hospital. The primary complaint of the patient was a severe chest pain lasting for $30 \mathrm{~min}$. When obtaining the medical history and initial examination, the patient appeared healthy and had no other complaints, diseases or known risk factors for coronary syndromes. The primary assessment of the attending physicians and management plan were focused on a musculoskeletal cause of the chest pain, taking into consideration the young age of the patient, regular physical training, and the lack of cardiovascular risk factors. The patient was discharged with oral acetaminophen to be taken when needed.

At home, the patient's pain increased in severity and was accompanied by excessive sweating and nausea. Consequently, his family brought him back to the central university hospital. The patient was admitted to the ED; he became dizzy, his blood pressure was $80 / 40 \mathrm{mmHg}$, his pulse was regular (pulse rate $101 / \mathrm{min}$ ), his respiratory rate was $20 / \mathrm{min}$ and his blood $\mathrm{O}_{2}$ saturation was $90 \%$. The patient's electrocardiogram (ECG) revealed ST-segment elevation up to $4 \mathrm{~mm}$ in leads V2 and V3.

At this stage, the diagnosis of a massive AMI associated with cardiogenic shock was established. Immediately, the patient was administered intravenous heparin $(5,000 \mathrm{IU})$, oral therapy with aspirin (300 mg crushed), clopidogrel (600 mg loading dose), atorvastatin $(40 \mathrm{mg})$, and provided with optimum oxygen supply (simple mask, 3 liters/min).

Blood chemistry was obtained and demonstrated increased levels of ischemic cardiac markers creatine kinase (CK) (10,387 U/l) and CK-MB (958.1 U/l). The fasting lipid profile revealed a very low concentration of high density lipoprotein (HDL) $(0.4 \mathrm{mmol} / \mathrm{l})$, with normal levels of low density lipoprotein (LDL) $(1.48 \mathrm{mmol} / \mathrm{l})$.

The patient was transferred to the cardiac care unit (CCU) for monitoring and preparation for urgent catheterization. In the $\mathrm{CCU}$, a new right bundle branch block was identified in the patient's ECG. The echocardiography demonstrated low ejection fraction (35\%), anterior apical wall akinesia with grade I mitral regurgitation and the left ventricular end diastolic diameter was $5.7 \mathrm{~cm}$.

Urgent cardiac catheterization was performed $1 \mathrm{~h}$ after admission to the CCU, and total occlusion of the ostium of the left anterior descending artery by large thrombus was observed (Fig. 1). Successful recanalization was performed with a bare metal stent (BMS) $(3.5 \times 23 \mathrm{~mm})$, and thrombolysis in myocardial infarction 2 flow was established. The patient was then administered tirofiban using percutaneous coronary intervention (PCI). The left circumflex artery was normal; however, notably, the right coronary artery (RCA) was affected: The pre-bifurcation was totally occluded by an embolized thrombus (Fig. 2). This may explain the changes in ECG observed in the CCU. Successful recanalization was performed with a BMS $(3 \times 18 \mathrm{~mm})$. An intra-aortic balloon pump was inserted, and the patient was administered a small dose $(5 \mu \mathrm{g} / \mathrm{kg} / \mathrm{min})$ of the vasopressor dopamine for 2 days.

The patient had an uneventful hospital course. The intra-aortic balloon pump was removed, and treatment with the vasopressor was stopped after 2 days. After 7 days, the patient was discharged with the following medications: Clopidogrel [75 mg per os (PO) once daily]; aspirin (100 mg PO once daily); lansoprazole (30 mg PO once daily); atorvastatin (40 mg PO once daily); spirolactone (25 mg PO once daily); carvedilol (3.125 mg PO twice daily); lasix (40 mg PO once daily); and enalapril ( $2.5 \mathrm{mg} \mathrm{PO}$ once daily).

After obtaining a detailed history, and when the patient realized the seriousness of his condition, he admitted that he has been using anabolic steroids and amino acids supplementations (sustanon $250 \mathrm{mg}$, once per week for 6 months and whey protein isolate, 6 tabs every day for 1 year).

During follow up, the patient continued to suffer heart failure with low ejection fraction. In addition, he developed an apical thrombus 2 months after admission. The patient developed tachycardia in spite of optimal medical treatment, and finally received an implantable cardioverter defibrillator.

\section{Discussion}

Few case reports of AMI occurring in healthy individuals who are regularly/chronically using AASs have been documented in the literature $(2,5-8)$. The lack of predisposing risk factors for AMI, young age, male sex, being physically active and the concurrent use of AASs were common factors in the majority of these previous reports. All of the reports concluded that the concomitant use of AAS may be responsible for these events, as a well-known cardio-toxic factor. However, none of these cases were able to prove a causal association between AAS overuse and IHD. In agreement with this observational association, evidence on other AAS-induced cardiovascular complications exist. For example; chronic AAS abuse has been associated with ischemic stroke and cerebral hemorrhage, renal infarction and sudden cardiac death (7,9-11).

In the present case report, although the patient was a physician, he did not realize the seriousness of his symptoms until his condition had deteriorated significantly. The patient did not disclose his chronic use of AASs, and the attending physician trusted the patient's reported history. At first admission, both the patient and the attending physician were considering a musculoskeletal cause of the chest pain. The present report emphasizes the fact that medical professionals can be AASs users and they may ignore this usage and its association with IHD. This may be associated with the effect of these substances on the users mental status (12), the common association between musculoskeletal chest pain and intense sports activities, or the embarrassment of the medical professional users to declare AAS usage, particularly because they are generally perceived to understand the catastrophic effects of AASs.

The potential underlying pathological processes that predispose individuals to AAS-induced AMI may include atherogenic, 


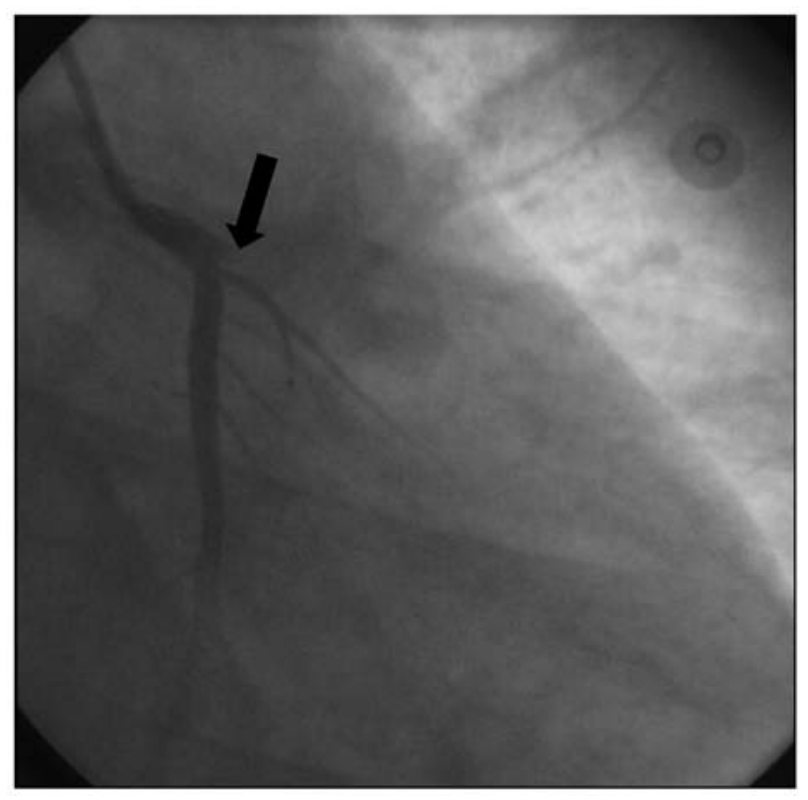

Figure 1. Angiogram of the left coronary branches. The black arrow indicates total ostial occlusion of the left anterior descending artery by large thrombus.

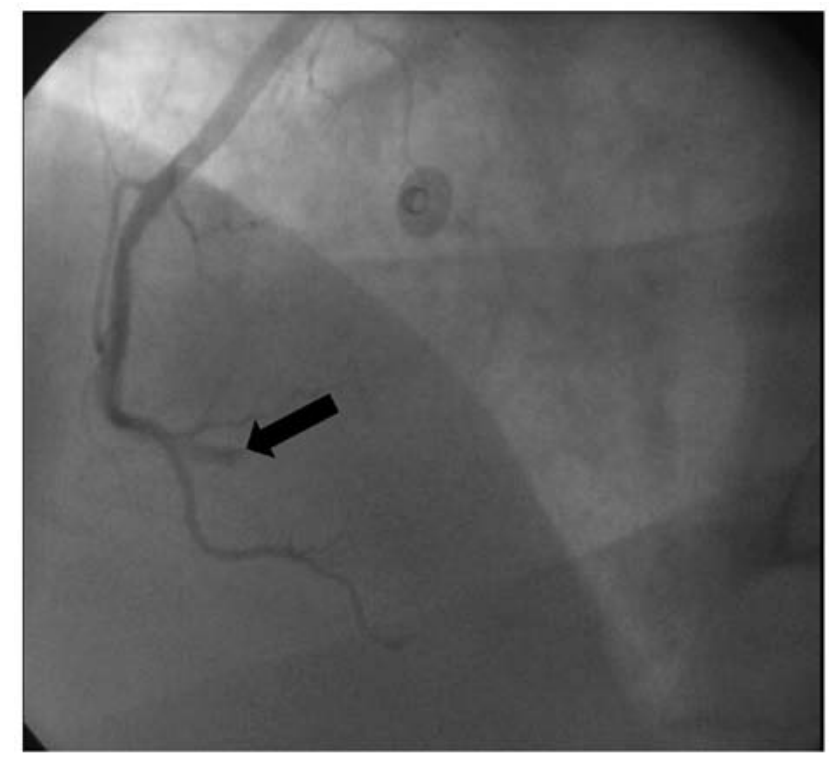

Figure 2. Angiogram of the right coronary branches. The black arrow indicates the area of the right coronary artery affected. The pre-bifurcation was totally occluded by embolized thrombus. thrombotic and vasospastic mechanisms, which were previously reviewed (5). The risk of thrombosis may be increased with AAS usage, which has been identified to activate platelet aggregation $(13,14)$. Conversely, AAS usage, particularly in high doses, is known to stimulate erythropoiesis, which in turn can increase blood viscosity and may predispose for vascular thrombosis (2). In the present report, the blood hematocrit values of the patient were normal. Although not extensively investigated, these normal values may negate such an assumption of erythropoiesis stimulation in this case. In addition, AAS abuse can change patient lipid profiles unfavorably; with increased levels of LDL-C and decreased levels of HDL and lipoprotein-A (9). This was confirmed in the present case report; however, in all previous case reports that documented these changes, the patients were not screened for a sufficient length of time. In these patients, the lipid values were measured at the time when the patients were admitted to hospital due to AMI symptoms.

Notably, in the present case, the patient may have suffered from both atherogenic (Fig. 1) and thrombotic (Fig. 2) coronary blockages. However, as it was not possible to examine the patient prior to hospital admission, it is unknown if the use of AASs caused the left atherosclerotic coronary lesion or, alternatively, if this use exacerbated a pre-existing lesion. Conversely, there was no clear evidence of an intra-ventricular thrombus, which may have explained the embolic thrombus in the right coronary artery. The documented evidence of the ability of AASs to increase blood thrombosis (5) may explain this event. In addition, although the vasospastic mechanism is less established as a factor in AASs-based AMI $(15,16)$, it may possibly intensify the atherogenic or the thrombotic mechanisms of AMI in AASs users. In concordance with these data, AAS abuse has been identified to induce vascular and endothelial dysfunctions $(17,18)$. Therefore, vasospastic agents were added to the pharmacological regimen of the patient in the present report. In addition, the ability of chronic AASs use to cause arrhythmias $(19,20)$ may lead to spontaneous formation of small thrombotic embolisms, which in turn, may be transported toward the coronary arteries and cause significant blockages. Notably, the AMI itself in chronic AASs users may also cause disruption to the autonomic nervous supply of the heart, which may predispose these individuals to spontaneous arrhythmias $(5,21)$. In turn, this may cause a thrombotic event within the ventricles, which may translocate to the coronary arteries and cause an embolism.

Conversely, it has been suggested that chronic AASs use may affect ventricular diastolic function (22). It may also cause significant hypertrophy and consequent arrhythmias (23). Coronary artery filling and normal coronary blood flow depends on the diastolic period of the cardiac cycle. Therefore, this may cause blood stasis, blood flow turbulence, and can affect the endothelial-vascular functions. Consequently, it may predispose patients to thrombotic or atherosclerotic events. The hypertrophic nature of the heart in AAS users can also contribute to this mechanism. In addition, certain studies have investigated the role of chronic AASs abuse in increasing both systolic and diastolic blood pressure values (24), which in turn may increase the heart load and predispose for AMI. However, the patient in the present report arrived at the ER in cardiogenic shock. Therefore, the chronic effect on blood pressure or cardiac hypertrophy were not measured, and remain unknown.

Finally, it was difficult to evaluate if the chronic complications in the patient were due to the AASs use or his acute ischemic condition. However, a number of previous studies in the literature describe the ability of AASs to affect the functions of the heart even after long periods of discontinued use (3-5).

In conclusion, a case of a massive anterior MI in a young physically active person who had no past medical history of any cardiovascular risk factors was described. In this case, AMI incidence was complicated with a cardiogenic shock and a mid-RCA embolic obstruction. The chronic use of AASs was considered as the most likely cause for the condition of the patient. 
Physicians should always be aware of the possibility of AASs use in young patients suffering from chest pain, particularly in patients without obvious risk factors for ischemic diseases. IHD should always be suspected and investigated with typical chest pain in healthy young patients, even if regular risk factors are not present. Increased efforts toward increasing public awareness of the potential complications of AASs abuse are required from medical and educational institutes. Medical professionals should not be excluded as potential AASs users/abusers.

\section{Acknowledgements}

Not applicable.

\section{Funding}

No funding was received.

\section{Availability of data and materials}

The ECGs and the records of the coronary angiogram are available from the authors upon reasonable request.

\section{Authors' contributions}

All authors contributed to the conception of the study. NA prepared the first draft and was a major contributor in writing the manuscript. MIJ and KHA revised the manuscript critically for important intellectual content. All authors read and approved the final manuscript.

\section{Ethics approval and consent to participate}

The publication of this report was approved by the local Institutional Review Board of King Abdullah University Hospital affiliated with the Jordan University of Science and Technology (approval no. 781182018). The requirement for patient consent was waived.

\section{Patient consent for publication}

The requirement for patient consent for publication was waived.

\section{Competing interests}

The authors declare that they have no competing interests.

\section{References}

1. Santos RP, Pereira A, Guedes H, Lourenço C, Azevedo J and Pinto P: Anabolic drugs and myocardial infarction-a clinical case report. Arq Bras Cardiol 105: 316-319, 2015 (In English, Portuguese).

2. Stergiopoulos K, Brennan JJ, Mathews R, Setaro JF and Kort S: Anabolic steroids, acute myocardial infarction and polycythemia: A case report and review of the literature. Vasc Health Risk Manag 4: 1475-1480, 2008.

3. Luijkx T, Velthuis BK, Backx FJ, Buckens CF, Prakken NH, Rienks R, Mali WP and Cramer MJ: Anabolic androgenic steroid use is associated with ventricular dysfunction on cardiac MRI in strength trained athletes. Int J Cardiol 167: 664-668, 2013.
4. Kersey RD, Elliot DL, Goldberg L, Kanayama G, Leone JE, Pavlovich $M$ and Pope HG Jr; National Athletic Trainers' Association: National athletic trainers' association position statement: Anabolic-androgenic steroids. J Athl Train 47: 567-588, 2012.

5. Christou GA, Christou KA, Nikas DN and Goudevenos JA: Acute myocardial infarction in a young bodybuilder taking anabolic androgenic steroids: A case report and critical review of the literature. Eur J Prev Cardiol 23: 1785-1796, 2016.

6. Peoples K, Kobe D, Campana C and Simon E: Hyperhomocysteinemia-induced myocardial infarction in a young male using anabolic steroids. Am J Emerg Med 32: 948. e1-2, 2014.

7. Ilhan E, Demirci D, Güvenç TS and Calık AN: Acute myocardial infarction and renal infarction in a bodybuilder using anabolic steroids. Turk Kardiyol Dern Ars 38: 275-278, 2010.

8. Wysoczanski M, Rachko M and Bergmann SR: Acute myocardial infarction in a young man using anabolic steroids. Angiology 59: 376-378, 2008.

9. Hartgens F, Rietjens G, Keizer HA, Kuipers H and Wolffenbuttel BH: Effects of androgenic-anabolic steroids on apolipoproteins and lipoprotein (a). Br J Sports Med 38: 253-259, 2004.

10. Fineschi V, Riezzo I, Centini F, Silingardi E, Licata M, Beduschi $G$ and Karch SB: Sudden cardiac death during anabolic steroid abuse: Morphologic and toxicologic findings in two fatal cases of bodybuilders. Int J Legal Med 121: 48-53, 2007.

11. Kennedy MC, Corrigan AB and Pilbeam ST: Myocardial infarction and cerebral haemorrhage in a young body builder taking anabolic steroids. Aust N Z J Med 23: 713, 1993.

12. Lyngberg KK: Myocardial infarction and death of a body builder after using anabolic steroids. Ugeskr Laeger 153: 587-588, 1991 (In Danish).

13. Pilo R, Aharony D and Raz A: Testosterone potentiation of ionophore and ADP induced platelet aggregation: Relationship to arachidonic acid metabolism. Thromb Haemost 46: 538-542, 1981.

14. Kokkonen L, Anttonen O, Penttilä O and Voutilainen S: Protein $\mathrm{C}$ deficiency and use of anabolic steroids behind the myocardial infarction in a young man. Duodecim 117: 2279-2281, 2001 (In Finnish).

15. Buttner A, Sachs H, Mall G, Tutsch-Bauer E and Weis S: Progressive idiopathic bilateral striato-pallido-dentate calcinosis (Fahr's disease) in a person with anabolic steroid abuse. Leg Med (Tokyo) 3: 114-118, 2001.

16. Sonmez E, Turkdogan KA, Yilmaz C, Kucukbuzcu S, Ozkan A and Sogutt O: Chronic anabolic androgenic steroid usage associated with acute coronary syndrome in bodybuilder. Turk J Emerg Med 16: 35-37, 2016.

17. Skogastierna C, Hotzen M, Rane A and Ekström L: A supraphysiological dose of testosterone induces nitric oxide production and oxidative stress. Eur J Prev Cardiol 21: 1049-1054, 2014.

18. Tischer KH, Heyny-von Haussen R, Mall G and Doenecke P: Coronary thrombosis and ectasia of coronary arteries after long-term use of anabolic steroids. Z Kardiol 92: 326-331, 2003 (In German).

19. Phillis BD, Abeywardena MY, Adams MJ, Kennedy JA and Irvine RJ: Nandrolone potentiates arrhythmogenic effects of cardiac ischemia in the rat. Toxicol Sci 99: 605-611, 2007.

20. Sculthorpe N, Grace F, Jones P and Davies B: Evidence of altered cardiac electrophysiology following prolonged androgenic anabolic steroid use. Cardiovasc Toxicol 10: 239-243, 2010.

21. Maior AS, Carvalho AR, Marques-Neto SR, Menezes P, Soares PP and Nascimento JH: Cardiac autonomic dysfunction in anabolic steroid users. Scand J Med Sci Sports 23: 548-555, 2013.

22. Kasikcioglu E, Oflaz H, Umman B and Bugra Z: Androgenic anabolic steroids also impair right ventricular function. Int $\mathrm{J}$ Cardiol 134: 123-125, 2009.

23. Frati P, Busardò FP, Cipolloni L, Dominicis ED and Fineschi V: Anabolic androgenic steroid (AAS) related deaths: Autoptic, histopathological and toxicological findings. Curr Neuropharmacol 13: 146-159, 2015.

24. Gheshlaghi F, Piri-Ardakani MR, Masoumi GR, Behjati M and Paydar P: Cardiovascular manifestations of anabolic steroids in association with demographic variables in body building athletes. J Res Med Sci 20: 165-168, 2015. 